\title{
Fortgeschrittenes Ovarialkarzinom: Schlechtere Prognose bei Gewichtsverlust während der CT
}

\begin{abstract}
Übergewicht gilt auch bei der Entwicklung eines epithelialen Ovarialkarzinoms (EOC) als Risikofaktor, aber die Gewichtsabnahme während der Chemotherapie ist dann meist ein schlechtes Zeichen: Eine retrospektive Analyse.
\end{abstract}

\begin{abstract}
n einer retrospektiven Analyse von 190 EOC-Krankheitsverläufen zwischen 2008 und 2013 wurde der Zusammenhang zwischen Gewichtsabnahme während der Erstlinienchemotherapie und Prognose überprüft. Die meisten Frauen hatten ein fortgeschrittenes High-gradeEOC und waren komplett oder bestmöglich reseziert worden. Während sich in frühen Stadien keine signifikanten Unterschiede im Körpergewicht im Verlauf der Chemotherapie zeigten, ergaben sich bei Frauen in fortgeschrittenen Stadien signifikante Schwankungen, die mit dem progressionsfreien Überleben (PFS) korrelierten. EOC-Patientinnen, die innerhalb der ersten sechs Monate mehr als $5 \%$ ihres Gewichts verloren oder mehr als $5 \%$ gegenüber ihrem ursprünglichen Körpergewicht zugelegt hatten, zeigten ein schlechteres bzw. längeres Überleben als
\end{abstract}

Patientinnen mit weitgehend konstantem Gewicht (6 vs. 13 vs. 15 Monate). Patientinnen im fortgeschrittenen Stadium zeigten darüber hinaus signifikante Differenzen beim Gesamtüberleben (OS) in Abhängigkeit von der Gewichtsveränderung - mit 24,3, 42,4 und 66,2 Monaten. In der multivariaten Cox-Analyse ergab sich eine signifikante Beeinflussung des PFS bzw. des OS durch Gewichtsveränderungen vom ersten bis zum sechsten CTZyklus (PFS: Hazard Ratio [HR] 0,97; 95\%-Konfidenzintervall [95\%-KI] 0,950,99; OS: HR 0,94, $95 \%$-KI 0,91-0,97). Das OS korrelierte darüber hinaus auch mit Gewichtsveränderungen vom dritten bis zum sechsten Zyklus (HR 0,93, 95\%-KI 0,88-0,98).

Fazit: Frauen mit einem fortgeschrittenen Ovarialkarzinom, die während der
Eine Gewichtszunahme ist erwünscht, wenn man eine Chemotherapie wegen eines Ovarialkarzinoms absolviert.

Erstlinienchemotherapie mehr als 5\% Gewicht verloren, hatten ein verschlechtertes PFS und OS. Eine Gewichtszunahme um mehr als $5 \%$ verbesserte die Prognose. Bei Patientinnen in frühen Stadien wurde dagegen kein derartiger Zusammenhang gesehen. Inwieweit diese Zusammenhänge von weiteren Parametern wie körperliche Aktivität oder Ernährung beeinflusst werden, konnte in dieser Beobachtungsstudie nicht ermittelt werden.

Barbara Kreutzkamp

Mardas M et al. Body weight changes in patients undergoing chemotherapy for ovarian cancer influence progression-free and overall survival. Support Care Cancer. 2017;25(3):795-800.

\section{MOGT Stadium I: Keine adjuvante Chemotherapie}

\section{Das Therapiemanagement bei malignen ovariellen Keimzelltumoren (MOGT) ist eher empirisch als evidenzbasiert. Daten einer retrospektiven Analyse geben jetzt weitere Anhaltspunkte für die Behandlung speziell im Stadium I.}

$\mathrm{D}$ as Standardvorgehen umfasst eine möglichst fertilitätserhaltende Operation inklusive Staging, gefolgt von einer platinbasierten Chemotherapie. Bei IA-Dysgerminomen oder unreifen Teratomen im Stadium IA/Grad 1 wird auf eine Chemotherapie meist verzichtet. Allerdings sind die Empfehlungen für diesen vergleichsweise selten vorkommenden Tumor noch nicht genügend evidenzbasiert. Die multizentrische Studie MITO-9 (Multicenter Italian Trials in Ovarian cancer) lieferte retrospektive Daten für das Vorgehen im Stadium I.

55 (38,2\%) der 144 Patientinnen hatten ein Dysgerminom, 49 (34\%) ein un- reifes Teratom, 26 (18,1\%) einen Dottersack- und 14 (9,7\%) einen gemischten Tumor. 71 Patientinnen (49,3\%) unterzogen sich lediglich einer Operation, 71 $(49,3 \%)$ zusätzlich einer Chemotherapie. 44 Patientinnen erhielten keine Chemotherapie, obwohl sie laut Leitlinien indiziert gewesen wäre.

Ingesamt 5 Patientinnen starben tumorbedingt, 4 davon mit einem Dottersack-Karzinom. Beim Gesamtüberleben (OS) erwies sich vor allem diese Histologie oder ein gemischter Tumor als prognostisch ungünstig - das 5-Jahres-OS betrug hier $83,7 \%$ gegenüber $98,6 \%$ bei anderen histologischen Typen $(\mathrm{p}<0,05)$.
Das 5-Jahres-OS lag nach operativem Staging bei $96,8 \%$ und nach inkomplettem Staging bei $88,7 \%$ sowie bei $93,8 \%$ nach Standardbehandlung im Vergleich zu $94,1 \%$ in der Beobachtungsgruppe. In der Cox-Regressionsanalyse war ein Rezidiv mit einem inkompletten operativen Staging assoziiert (Odds Ratio 2,37; $\mathrm{p}<0,05)$.

Fazit: Patientinnen mit MOGT im Stadium I benötigen in der Regel lediglich eine Operation, die zusätzliche Chemotherapie verbessert die Prognose nicht und sollte Rezidiven vorbehalten bleiben. Ein inkomplettes chirurgisches Staging ist mit einer höheren Rezidivrate assoziiert, eine Dottersack-Histologie mit einem verschlechterten 5 -Jahres-OS.

\section{Barbara Kreutzkamp}

Mangili G et al. The role of staging and adjuvant chemotherapy in stage I malignant ovarian germ cell tumors (MOGTs). Ann Oncol. 2017; 28(2):333-8. 